This item was submitted to Loughborough's Research Repository by the author.

Items in Figshare are protected by copyright, with all rights reserved, unless otherwise indicated.

\title{
Applications of converged networks in construction
}

PLEASE CITE THE PUBLISHED VERSION

PUBLISHER

(C) Inderscience Enterprises Ltd

VERSION

AM (Accepted Manuscript)

LICENCE

CC BY-NC-ND 4.0

REPOSITORY RECORD

Ahsan, Shabbir, Dino Bouchlaghem, Ashraf El-Hamalawi, and Salman Ahmad. 2019. "Applications of Converged Networks in Construction". figshare. https://hdl.handle.net/2134/5156. 
This item was submitted to Loughborough's Institutional Repository (https://dspace.lboro.ac.uk/) by the author and is made available under the following Creative Commons Licence conditions.

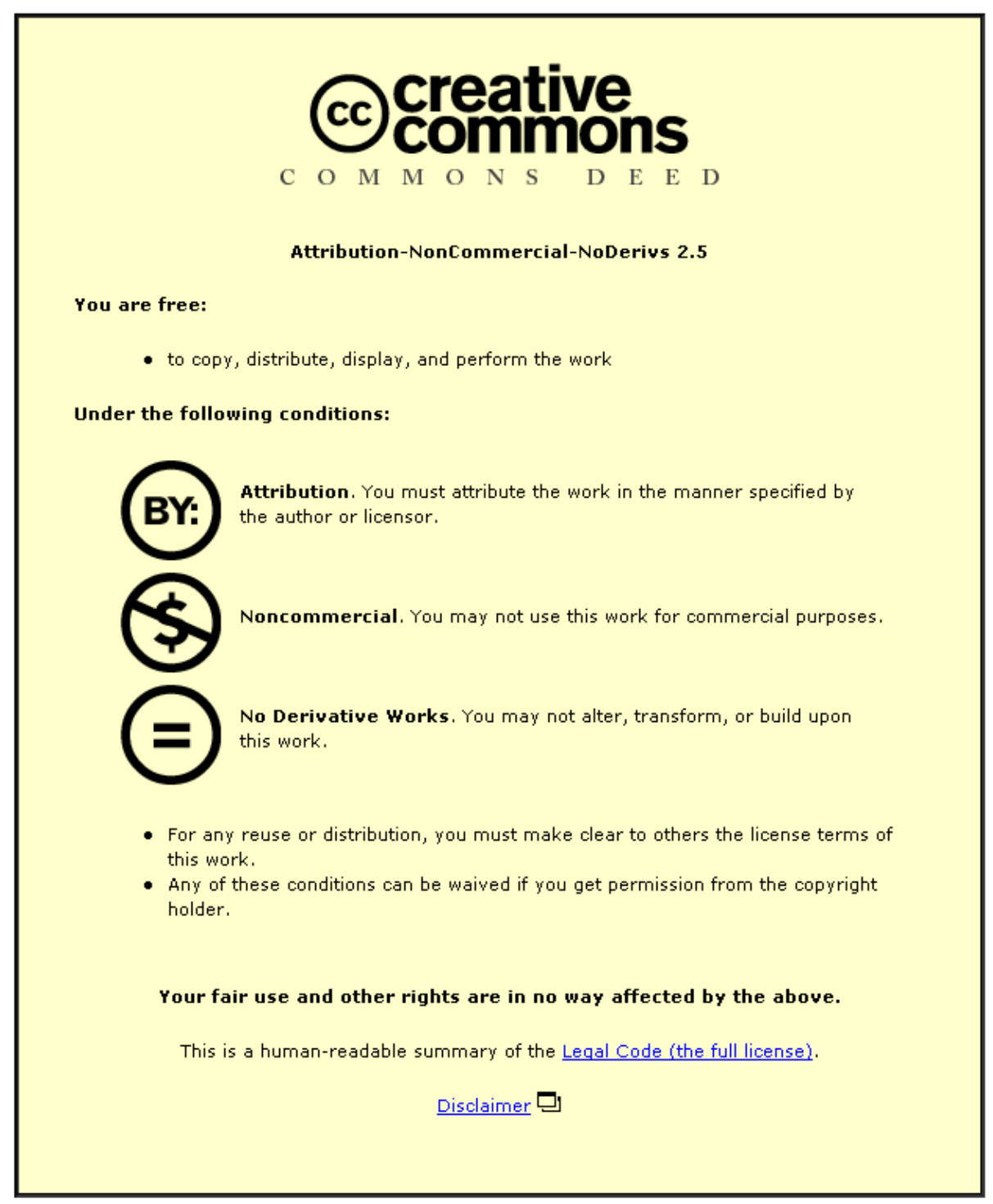

For the full text of this licence, please go to: http://creativecommons.org/licenses/by-nc-nd/2.5/ 


\title{
Applications of Converged Networks in Construction
}

\section{S. Ahsan, A. El-Hamalawi, D. Bouchlaghem \& S. Ahmad}

\author{
CICE, Loughborough University, Loughborough, LE11 3TU, UK \\ Email: M.S.Ahsan@lboro.ac.uk \\ Email: A.El-hamalawi@lboro.ac.uk \\ Email: N.M.Bouchlaghem@lboro.ac.uk \\ Email: Salman.Ahmad@myriadvision.com
}

\begin{abstract}
Converged networks which carry voice, video and data have promised cost savings for its users. Much of the construction industry spends over $£ 1$ million on communication, mainly from the construction sites to headquarters. With the use of IP telephony and converged networks, the cost can be reduced significantly. Using existing $802.11 \mathrm{~b} / \mathrm{g}$ wireless networks, access to the voice network can be delivered on a construction site allowing mobile users access to personnel as well as data instantly.

Voice over Internet Protocol (VoIP) plays a major role as a catalyst for convergence. Although it is sometimes viewed as just another means of transporting voice communication, the use of one single network provides opportunities for advanced applications that can support and enhance the construction workflow. The introduction of Voice over a Wireless Local Area Network (VoWLAN) allows the opportunities to expand onto the construction site.

This paper looks at the emergence of VoIP and examines how this can be brought to the construction site. The paper reports on research carried out with the UK construction industry, identifying communication trends as well as information storage of this communication. With this information, the paper attempts to identify processes where the technology can provide key benefits
\end{abstract}

Keywords: VoIP, VoWifi, Construction Communications, Project Management, Voice control

Reference to this paper should be made as follows: Ahsan, S., El-

Hamalawi, A., Bouchlaghem, D and Ahmad, S. (xxxx)'Applications of converged networks in construction’, Int. J. Product Development, Vol. X, No. Y,pp.000-000.

\section{Introduction}

Communication is at the core of the majority of processes within the construction industry. The main reason for this is that the industry relies heavily on a team structure. Individuals or teams are usually composed of heterogeneous and fragmented firms which are different in terms of size and scope of work (Anumba et al., 1997). 
Ahsan S, El-Hamalawi, A., Bouchlaghem, D. and Ahmad, S.

Communication therefore lies at the heart of the team achieving its objectives successfully. In order for the construction team to achieve its task efficiently, constant communication between the construction and design team is crucial. The time taken to access information and personnel is a factor in productivity of the construction team (Miah et al, 1998); however the necessity for constant communication can lead to a large overhead on the construction project. The move to a unified network could reduce some of these overheads by allowing users access to both data and personnel via voice, video and data communication on a single network that is controlled and established by the construction team themselves, thus allowing for instant access and control of costs.

A look at the different communication methods used within construction can identify the telephone as either the primary or the secondary method used (Howard and Petersen, 2001). Telephone technology has advanced within construction to embrace mobile telephony due to the advantages of mobility. This flexibility however comes with a heavy price tag, which the surveys have identified. Other communication technologies have been tested on site, such as the DECT (Digital Enhanced Cordless Telecommunications) phone (Meissner et al., 2001), which has proved useful, however these technologies are not suitable for converged applications.

Voice over Internet Protocol (VoIP) is the process of digitalising and sending voice traffic over a data network as opposed to the time-division multiplexed (TDM) method used on traditional phone networks (Wallingford, 2005). The process has become increasingly used to send voice traffic over the internet, bypassing the traditional telephone network and allowing for voice communication to occur with no charge. The use of internet protocols remove the barriers traditionally set on a voice network, allowing for the benefits to be extended to remote offices, suppliers and partners (Thus, 2006). The adoption of VoIP has seen increasing interest in the corporate sector due to the potential for large cost savings, with an extended feature set (AT \& T, 2004). Running voice communication on a unified network can bring about cost savings; however the possibility for enhanced, integrated applications can bring about greater possibilities for cost savings and give a competitive advantage to its users.

With the introduction of WiMAX (Worldwide Interoperability for Microwave Access), a standards-based wireless technology that provides high-throughput broadband connections over long distances (www.wimax.co.uk, 2006), the ability to send voice and data over a wireless network is improved. Trials with sending voice and data over a WiMAX network have shown that the infrastructure is suitable (Agapiou, et al., 2006). WiMAX technology will initially be used as a 'last mile' broadband delivery, and can allow access to broadband from remote areas, or areas where a cabling infrastructure does not exist, but as more WiMAX clients are introduced, the technology will be used directly by consumers. This therefore allows for the construction site to be connected to the internet without the need of a hard wired connection.

In a recent survey carried out by AT\&T of 236 executives, it was found that sixty percent of companies will have deployed converged networks across most of their organisations by 2008. The survey identified that there was an important shift in the emphasis of network convergence from cost-cutting to value-creation (AT\&T, 2005). Researchers have identified some advantages of convergence as (Corte, A., et al., 2006):

- Scalability and flexibility

- Availability

- Easy management and configuration of users and network devices 


\section{Applications of converged networks in construction}

- $\quad$ Increase in productivity.

Of the organisations which have embarked on full scale convergence implementation, many have found the immediate advantage offered include cost-savings between their various sites, the ability to easily augment networks and resources from different sites, and reduced time to access important information (Cable \& Wireless, 2001).

In this paper the converged network in respect to construction will be examined. The general requirements of the industry and industry perceptions will be examined along with potential deployment scenarios. The use of deployments scenarios along with existing case studies will be used to put the case forward for the construction industry to adopt the new technology.

\section{Construction Communication and Collaboration}

Communication within construction has always been important due to the nature of work involved. However the introduction of more inclusive project methodologies has ensured the necessity for communication to span across the entire project lifecycle and across all disciplines involved.

\subsection{Collaborative Engineering}

The speed at which the project models have been put forward have been enhanced with the uptake of an efficient project methodology, concurrent engineering, now more commonly known as Collaborative Engineering. Collaborative engineering (CE) was introduced successfully in the manufacturing industry, initially as a means to bring down the product design development and delivery (DARPA, 1987), but many definitions emerged. The many definitions still exist today as the concept is being transferred into the construction industry. $\mathrm{CE}$ is understood to be a parallel approach, replacing the traditional sequential "over the wall” process.

$\mathrm{CE}$ has been described as "the methodology encompassing the framework for resolving the problems arising from the sequential approach. It encourages team working, consisting of multi-disciplinary personnel from all the major departments such as engineering, manufacturing, purchasing, sales, and support, quality and so on. These teams carry the full responsibility for new products from concept through to manufacture.” (Syan, 1997). “CE embodies methodologies such as multi-disciplinary teams, parallel scheduling of activities and cross-functional problem solving” (Anumba, 1997). In the construction industry, the cost of making alterations after the completion of a project is extensive. There is therefore a huge emphasis on "getting it right" the first time. The move to CE comes with this realisation that it would help in producing a final product with minimum defects, and matching the user's requirements.

Although CE aims to achieve zero defects with the use of careful planning, many different aspects within the project change, and require a swift response. The UK construction industry has defects which cost at least $£ 20$ billion to repair or rebuild (BRE guidance, 2005). Poor communication on the construction site is the cause of some of these defects, with poorly detailed drawings, operatives being given incorrect instruction or technical information not being available all factors to this cost. Access to project 
Ahsan S, El-Hamalawi, A., Bouchlaghem, D. and Ahmad, S.

resources such as team members and project data is increasingly important to cut these losses.

\subsection{Construction Communications}

The research on construction communication practices was carried out with the UK construction industry with the use of surveys and interviews. The surveys targeted construction companies within the midlands with a $20 \%$ response rate. The survey was an online survey, and emails were sent to 90 individuals across the UK. A total of five follow up interviews were undertaken with managers of major UK construction companies including Marriott Construction, SOL Construction and Scott Wilson. The survey was used to understand the current practices, preferences, and technology used onsite. The surveys and interviews also explored the storage of communication from a project that would be used for later analysis.

Table 1 lists the results of communication preferences. The respondents were asked which form of communication they preferred to be contacted on and which they preferred to contact others on from a scale of 1 to 6 , with 6 being the least preferred and 1 being the most preferred. The research identified the telephone as the most preferred method of contacting other members using some sort of technology. Earlier surveys (Ahsan, et al., 2004) identified face to face communication as the ideal form of communication as it allowed for communication on more than one level.

Table 1 Communication Preference in Construction

\begin{tabular}{lll}
\hline Rank & Outgoing Communication & Incoming Communication \\
1 & Telephone & Mobile Phone -Call \\
2 & Mobile Phone -Call & Telephone \\
3 & Email & Email \\
4 & Mobile Phone -SMS & Mobile Phone -SMS \\
5 & Instant Messenger & Instant Messenger \\
6 & Walkie Talkie/Radio & Walkie Talkie/Radio \\
\hline
\end{tabular}

It is worth noting that in the interviews carried out after the surveys, it was expressed that the method of communication depended on the situation and where the individual was located. For example, on the construction site if it was a simple case of getting hold of someone, the radio was the device used.

The mobile phone appearing in second place for outgoing and first place for incoming communication shows the increased importance in mobility, and the reliance on the mobile phone. This corroborates previous research by Howard and Peterson in 2004 which was carried out in Denmark highlighting the similarities between the UK and international construction processes. The reliance on voice communication through these technologies falls in line with the amount spent on communication. The research showed that a majority of the industry spent over $£ 500$ thousand a year on communication, with most spending millions of pounds. This was however spent on communications for a number of projects. 
Applications of converged networks in construction

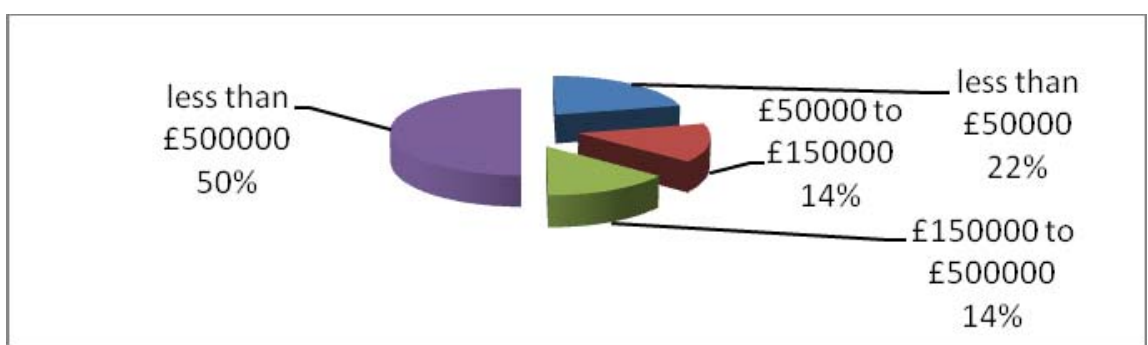

Figure 1 Pie Chart showing the cost of communications in construction companies for 2005

The research investigated the use of technology, in particular the use of wireless networks, and whether these were found to be of use, and where in the construction life cycle the technology was required. It was shown that $56 \%$ of respondents had a wireless network setup for employees, whereas $50 \%$ had the use of wireless networks on the construction site itself. The industry, however, responded with $92 \%$ wanting a wireless network on the construction site showing the increased awareness of the technology and the benefits it can bring.

Many different wireless technologies have been tested on construction sites including different infrastructures and communication devices (Bowden, 2002, Meissner et al., 2001, Beyh et al., 2004 and de la Garza et al., 1998). The Construction Site Mobile Operations Support (COSMOS) (Meissener et al., 2002) and Mobile Integrated Communication in Construction (MICC) (Deguine et al., 1999)projects tested various technologies on the construction site including DECT (Digital Enhanced Cordless Telecommunications), GSM (Global System for Mobile communications), WLAN (Wireless LAN), and TETRA (Terrestrial Trunked Radio). The MICC project identified the DECT technology to be suitable for the construction sector due to its reliability (Deguine et al., 1999). However the DECT system has problems with standards and cannot match the bandwidth of other technologies such as the wireless LAN, therefore running both data and voice intensive applications would prove difficult.

When identifying the various communication methods available, researchers have identified certain characteristics which should be looked into (Beyh et al., 2004):

- Benefits in terms of the nature of information that need to be transmitted including voice, data, video, web collaboration, etc.

- Access to all members forming the project teams including site workers, gangers, and foremen

- Reliability, availability and quality of service

- Cost of service including network administration, maintenance and upgrade

- Availability of terminals and users' devices such as mobile handsets.

Many of these technologies have been reviewed with an emphasis on data communications (Bowden, 2002, Meissner et al., 2001, de la Garza et al., 1998, CusBabic et al., 2000). Data communications occuring on the construction site usually require less bandwidth than voice communications and are less demanding on network resources. The wireless LAN proves to be of relative simplicity and provides the correct infrastructure for carrying voice and even video. The impact of such a system is highlighted as it could allow access to information and contact with remote experts from a construction site (Miah et al., 1998). 
Ahsan S, El-Hamalawi, A., Bouchlaghem, D. and Ahmad, S.

The research examined the storage of communication produced during a project. It was shown that the current practice was to store all printed documentation and email correspondence. The result of email appearing as the second most collected form of communication in this study lends more credibility to the fact that email has become increasingly important for communication within construction. Email lends itself particularly well to storage as all emails regarding a project can be extracted and saved.

Voice communication featured on a very small scale in the conducted study. However upon further investigation it was clear that the reason for this was due to the complexity of recording such a medium. The desire to record such communication was expressed, but the difficulty lies in the way voice communication is currently carried out - over varying networks such as the PSTN (Public Switched Telephone Network), or GSM. Although the complexity is currently withholding this requirement, the introduction and use of a converged network can allow it to become reality. Call logging and recording applications can easily be augmented onto the network as all voice would pass through a central area. The logging application would be able to log who was involved in a conversation with the recording application storing the conversation itself.

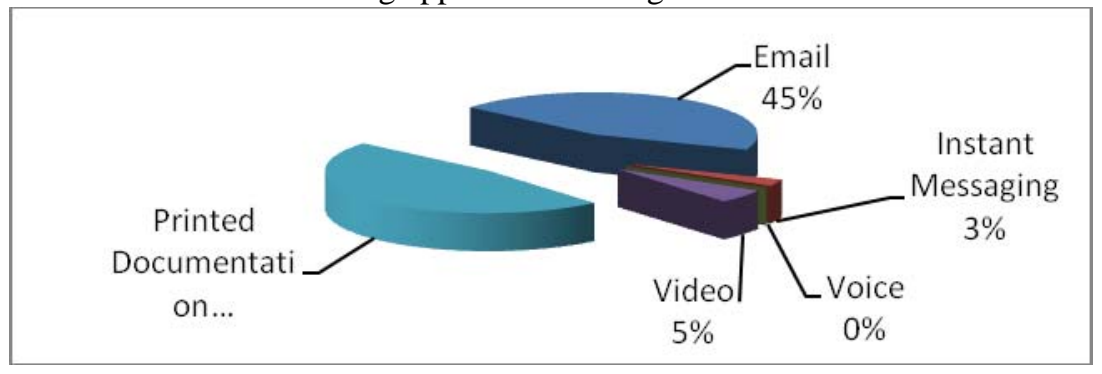

Figure 2 Stored Communication Media within UK Construction firms

\section{Voice over Internet Protocol}

In order to support the successful deployment of VoIP, the use of standards is crucial. The industry has adopted the session initiation protocol (SIP) because of its scalability, compatibility and ease of use (Prasad et al., 2003). The use of standards provides the platform for interoperability between equipment and software from different vendors.

For VoIP to be technically, economically and operationally feasible, the cost savings must be sufficient to justify the investment concerned and the payback on the investment should be within a realistic time span given the technology involved and the business environment concerned (Baldwin et al., 1994). It has been estimated that for the construction industry to implement a full scale conversion of IP telephony, the payback would take 2.06 yrs (BT, 2006). Full scale conversion includes the complete replacement of the traditional phone infrastructure with IP telephony. 


\subsection{VoIP Technology}

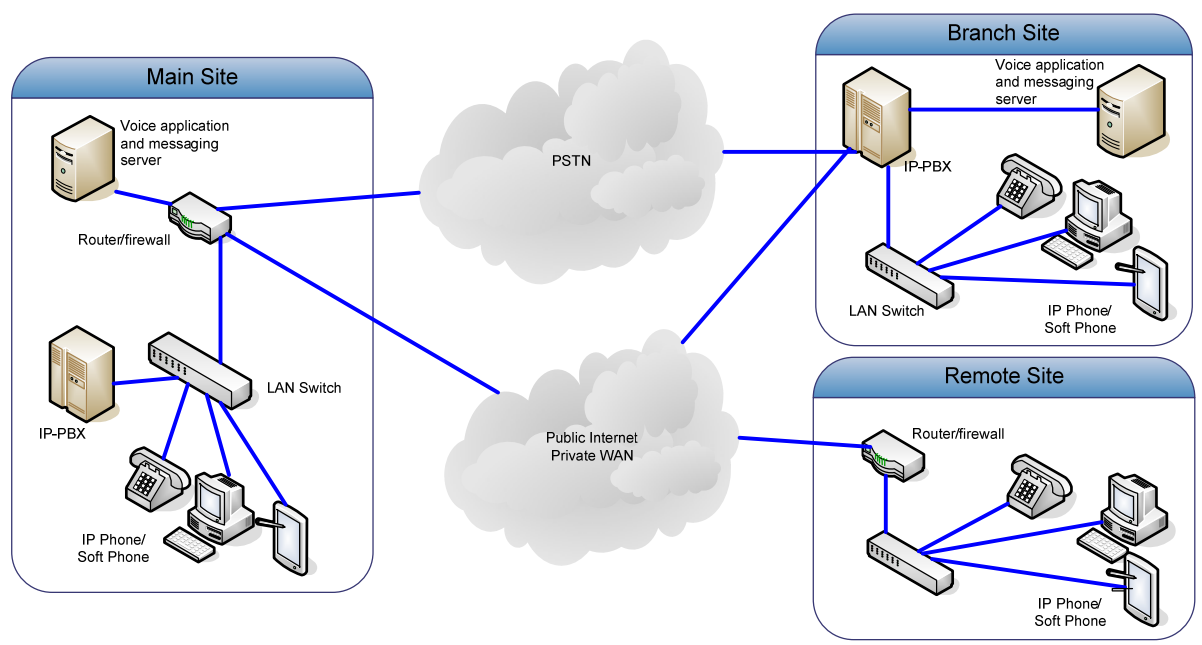

Figure 3 VoIP in the Enterprise (Thus, 2006)

Figure 3 is a typical VoIP deployment model where three sites are connected. The basic setup for the one site includes an IP-PBX, a network and client devices. The client devices can be either hardware phones or software based 'soft-phones'. When connecting different sites together over the internet or a private WAN, the need for routers capable of delivering quality of service (QoS) is important to deliver a reliable call service.

The deployment of VoIP technology into the construction industry would be very similar to the typical deployment. The remote sites in this case would be the construction sites themselves, with the deployment on the sites remaining for the duration of the project. The requirement would therefore be a setup which can easily be torn down and then rebuilt on different locations.

In order for communication between the construction site and head office to utilise VoIP, a method of connecting the two sites is required. Connection to the internet can be provided by broadband suppliers. However the use of leased lines can provide effective bandwidth. The advent of WiMAX would enhance the availability of broadband access on construction sites as physical lines would no longer be necessary.

\subsubsection{IP-PBX}

The IP-PBX is the IP version of the traditional PBX (Private Branch Exchange). The traditional PBX has 3 distinct functions: 1) call processing; 2) call switching; and 3) establishing trunk connections. These distinct functions can be translated into the IP alternative with relative ease.

Software based IP-PBX's are available including open source versions. Vendors are however releasing all-in-one devices containing the hardware and software to provide all the functionality including wi-fi to allow a smooth transition to voice over wi-fi. This type of system is potentially suited for the construction site where the requirement for speed and ease of deployment are high priorities. Most of these systems offer connection to other call management systems and can augment to an existing network allowing connection with the company head quarters. The drawback with such a system is that 
Ahsan S, El-Hamalawi, A., Bouchlaghem, D. and Ahmad, S.

most vendors limit the number of users that can connect. The open source versions such as Asterisk do not restrict the number of users that can connect, allowing for a greater degree of flexibility. Open source systems can also be setup to match and exceed the hardware specifications of the all-in-one solutions as hardware can easily be augmented onto the computer running the IP-PBX. The disadvantage of setting up an open source system to match the abilities of an all-in-one solution is the complexity of setup.

Deployment of an IP-PBX can follow two separate strategies. The two strategies which are emerging are the choice of internal implementation or using hosted environments. The hosted PBX is the outsourcing of communication to an external vendor. This has the advantage of a much quicker time to deployment and also a greatly reduced initial deployment cost. The cost of maintenance is also no longer required inhouse but managed by the company hosting the communications network. The disadvantage however, lies in the network being managed by a third party, rendering the use of convergence applications impossible as the voice traffic is running on a separate network and cannot communicate with data applications in external sites.

Most IP-PBX's will run using the Session Initiation Protocol (SIP). Some of the key advantages of SIP are as follows (Melinat \& Kelly, 2002):

- SIP is an Internet Protocol, it can facilitate the integration of communication applications with other web-based applications.

- SIP does not rely on any type of network.

- Using SIP, new applications can be easily added without the need to rely on one specific vendor.

\subsubsection{Router/Network}

Traditional data on a network can be subject to many disturbances therefore leading to delays in the data arriving at the destination. For data applications, these delays can be tolerated, however when voice is introduced these delays will not be accepted. With the adoption of VoIP, the traffic on the network would also increase and thus impact on the network performance. Most modern day networks are capable of handling the extra voice traffic. Most deployments of VoIP begin with a survey of the network to identify any potential bottlenecks and ensure the smooth flow of voice data. The identification and removal of bottlenecks will result in an efficient network that would benefit not just voice, but data users.

Several aspects have been identified as important by the VoIP industry with regards to the network. These are: 1) IP Precedence; 2) Quality of Service (QoS); and 3) Power over Ethernet (Wallingford, 2005). With these addressed, the network would perform under the pressures of extensive voice and video data. As most modern networks are capable of handling the extra load produced by voice and video data, the requirement for QoS may not be as important, however when linking offices together over unknown networks such as the internet, QoS becomes very important to ensure smooth call operation.

\subsubsection{Wireless Network}

The wireless network is essentially an extension of the wired network. The wireless network has gained increased popularity as it provides the benefits of being connected to a network without the hassle of any wires, allowing the user mobility. The wireless 


\section{Applications of converged networks in construction}

network works with radio waves, and there currently exist three popular standards produced by the American Institute of Electrical and Electronics Engineers (IEEE) which are in use today. The main difference in these three standards is the bandwidth they offer. The IEEE 802.11b was the first to have widespread adoption and offers a bandwidth of $11 \mathrm{Mb} / \mathrm{s}$. This has been updated with the IEEE 802.11g standard which manages to reach $54 \mathrm{Mb} / \mathrm{s}$ at the same frequency of $2.4 \mathrm{GHz}$. Some vendors have managed to increase this bandwidth utilising their own propriety software. The IEEE 802.11a standard offers the $54 \mathrm{Mb} / \mathrm{s}$ but at a higher frequency of $5 \mathrm{GHz}$. This has the advantage of a less crowded airspace providing a much more stable connection.

The wireless network can extend across large areas by bridging many network access points together. Different types of networks that use the same basic protocols can be augmented together allowing for data to pass seamlessly between them. There is an issue with handover, when passing from one access point to the next. This has been addressed by many network vendors with solutions that vary from dumb access points through to intelligent access points, and the use of a mesh structure instead of the traditional cell structure.

The technology considerations for the construction industry are unique as the technology has not only to work within an office environment, but on a construction site, which can limit the effectiveness. Wireless networks have been tested on construction sites primarily for data exchange. One such test ran with tablet PCs on a construction site connected as thin clients via a wireless network (Ward et al. 2004). The wireless network on a construction site can be set up relatively easily, and is scalable to cover large distances. The network can be set up to mimic the traditional cellular network, with access points marking the centre of each cell. This way, the network could be enlarged with an increased number of access points being 'daisy-chained' together as shown in figure 4 (De la Garza et al., 1998, Ward et al., 2004). Installing a wireless network on a construction site is very different to a traditional office. The outdoor environment has a major impact, with the effects of weather and dust which mean that the wireless technology should be rugged enough to cater for that.

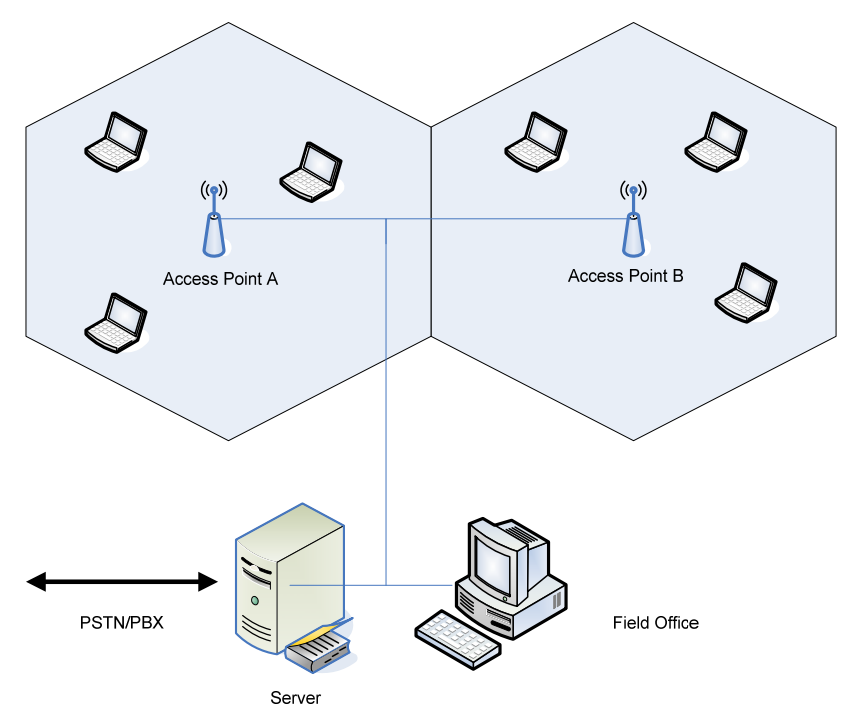

Figure 4 A WLAN network with access points (De la Garza et al., 1998) 
Ahsan S, El-Hamalawi, A., Bouchlaghem, D. and Ahmad, S.

With tests of wireless networks on construction sites, the researchers have used standard wireless equipment in rugged containers to keep them secure in the construction environment. Manufacturers of wireless equipment have developed systems specifically for the construction industry that are capable of withstanding the conditions on site. These systems can be installed and daisy-chained like ordinary wireless systems which have been identified. Figure 5 shows an example of such equipment. This specific device works with the $802.11 \mathrm{~b}$ standard with a maximum throughput of $22 \mathrm{Mb} / \mathrm{s}$ and a claimed range of up to 10 miles (CII).

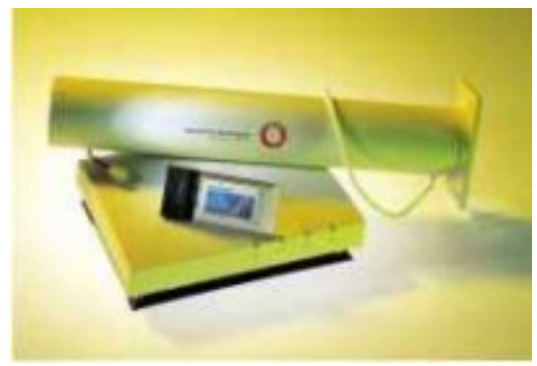

Figure 5 Orinoco Outdoor Router (CII)

Issues identified with the wireless setup included obtaining power for the wireless access point. This was resolved by connecting the access point to a rig's power supply, therefore bypassing the need for a battery (Ward et al., 2004). Device battery life has been identified as a problem with measures to reduce battery consumption including placing the device on standby.

The placement of the wireless access points can also provide some difficulty on a construction site. An American construction company, Webcor, installed a wireless access point on the crane hovering over the construction site. This wireless network gave engineers and crews access to blueprints and the ability to easily coordinate projects (Shim, 2004).

A potential drawback with delivering voice over a wireless network is within the 802.11 standard. The standard does not contain any QoS features resulting in the wireless infrastructure unsuitable for large volumes of calls. Some authors have suggested the wireless network to be unstable for voice use, even with the use of compressed codecs (Wallingford, 2005).

Transmitting voice over a wireless network, although a very new technology with many sceptics, has had success in the indoor market. Companies such as Vocera are supplying hospitals in America allowing for hands-free instant communication amongst hospital staff. The Vocera network utilises the $802.11 \mathrm{~b}$ standard for the wireless network, and using compressed codecs for the voice data is able to achieve a satisfactory level of service. This setup is unique as the company has produced specific communication devices which allow for one-touch calling (Vocera, 2004). The Vocera solution also encompasses voice recognition. This is necessary for one-touch dialling, whereby the user asks for a particular person or job role, the server then interprets this and connects to the resulting person. This technology could be applied in the construction field, although one-touch dialling may prove to be difficult to work with on a noisy construction site. Figure 6 shows the network structure with the Vocera solution. 


\section{Applications of converged networks in construction}

The diagram highlights the ability to link the voice solution with other applications, as all data is run over the same network using common standards. This is the case with any VoIP solution. The power of the VoIP solution is therefore enhanced with the ability to converge it with other applications to enhance collaboration.

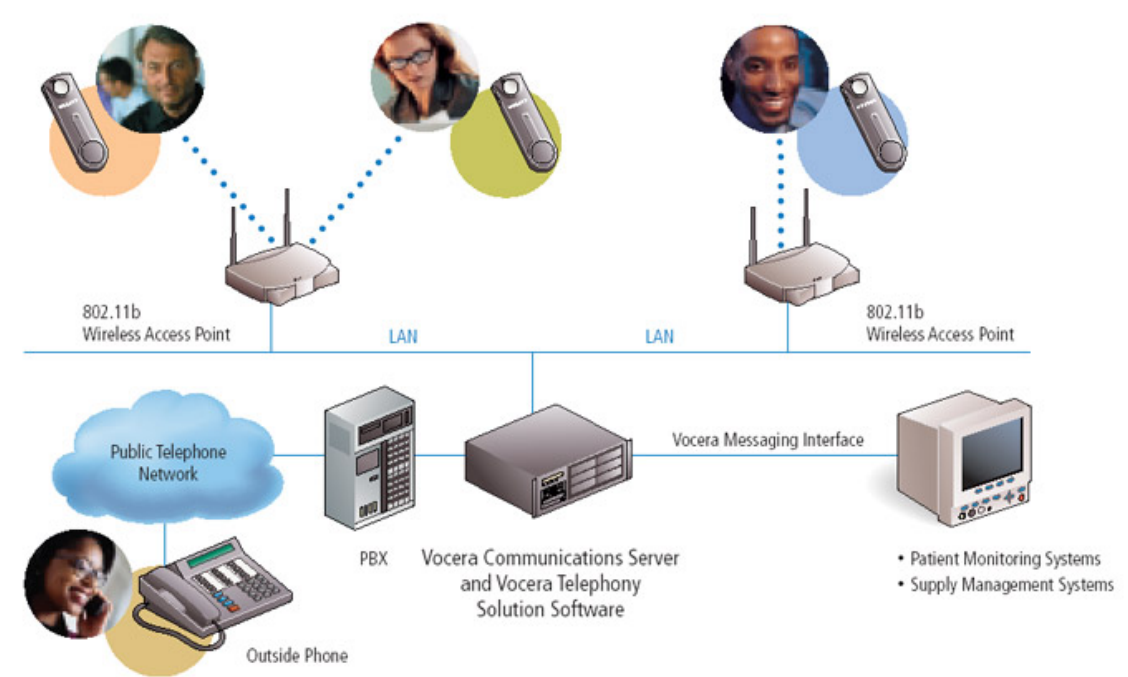

Figure 6 Vocera Networks Solution (Vocera, 2004)

Access to a wireless network has expanded with WiMAX due to be introduced into the commercial sector. WiMAX 802.16-2004 is a further advancement on the current 802.11 wi-fi standard offering fixed broadband wireless access (IEEE). The technology will be a significant enhancement allowing for broadband speeds of $15 \mathrm{Mbps}$ for a $5 \mathrm{MHz}$ channel to $35 \mathrm{Mbps}$ for a $10 \mathrm{MHz}$ channel, and ranges of kilometres (WiMAX Forum, 2005). The 802.16e standard will enhance the current 802.16-2004 standard to allow for roaming instead of fixed devices which will broaden the mobile internet market much further. Advantages of the new WiMAX standard including speed, distance and QoS provide fertile ground for further enhancing the convergence space. Some observers believe WiMAX poses a genuine threat to $3 G$ networks, although the two technologies are as much complementary as they are competitive (Ankeny, 2005).

\subsubsection{Client Device}

There are various client devices available to use ranging from hardware specific such as the various Cisco IP phones and the Vocera one-touch handsets through to software phones known as 'soft-phones'. Soft-phones can run on a computer as well as PDAs allowing the use of a PDA or pocket computer as a VoIP phone. The use of wireless networks and wireless PDAs enables the use of the PDA as a mobile VoIP handset.

Handset manufacturers including Nokia and Motorola have produced mobile handsets that are wi-fi compatible (Nokia N80, Nokia E60, Nokia E61, I-mate K-JAM), with the Nokia handsets preloaded with soft-phones. The aim is to allow seamless transfer of communication from a GSM network to the wi-fi network when it becomes available. 
Ahsan S, El-Hamalawi, A., Bouchlaghem, D. and Ahmad, S.

\subsection{Construction Industry perception of VoIP}

The research investigated the industry's perspective on VoIP technology, having discovered that $67 \%$ understood the term VoIP. This was mainly through home use with the use of software such as Skype which offers users PC to PC calls for free.

VoIP was understood by many respondents of the questionnaire as a means to save on communication costs, with the vast majority of respondents indicating the success of VoIP within construction lied in its support of communications between various offices. It is interesting to note that the ability to keep an audit trail, and the ability to use features such as click to call were identified by some respondents indicating an understanding of the capabilities of a converged network, and the benefits it can bring.

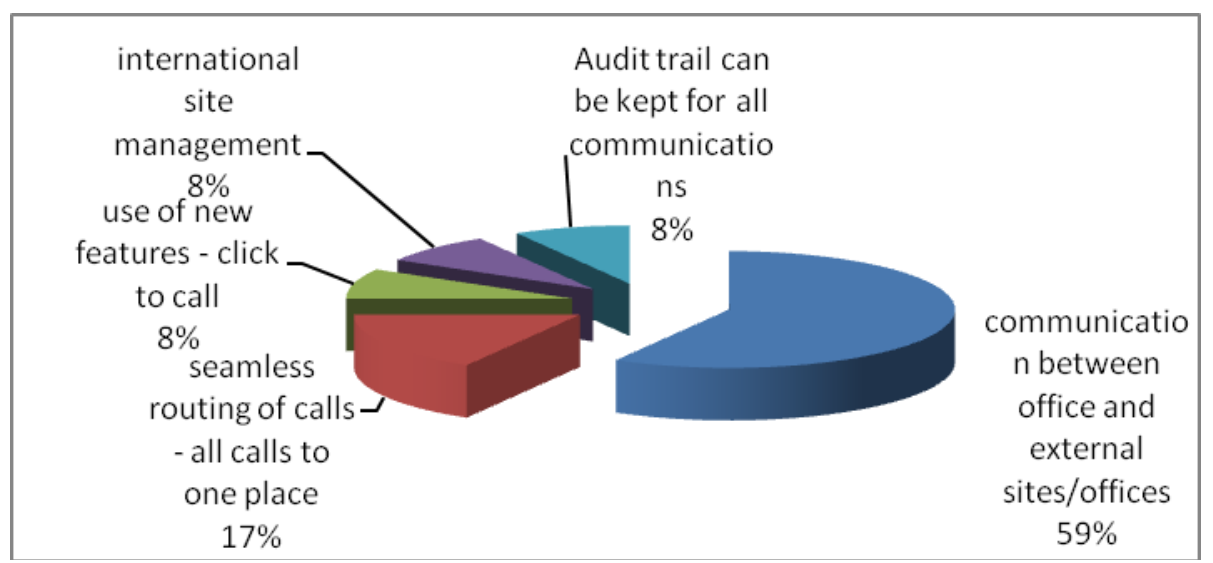

Figure 7 Where VoIP is Perceived to Work Best

The construction industry still remains cautious about the technology as it is still very new within most industry sectors. Surveying the perceived problems, setup costs, reliability and voice quality were identified as the largest issues. Issues of cost are valid, as the implementation of a converged network running voice, data and video would require investment, however a study of the costs of a VoIP setup found the cost significantly cheaper than that of a traditional phone setup in all aspects of cabling, deployment, training, management, maintenance, telephone traffic and interconnection apart from data/voice devices, i.e. the cost of IP phones (Corte, A., et al., 2006). The reliability of the converged network and the quality of voice calls are issues across all sectors where the technology is deployed. Most modern network hardware is capable of handling the increased bandwidth usage brought about with data, voice and video all running on the same network as most implement QoS protocols. 
Applications of converged networks in construction

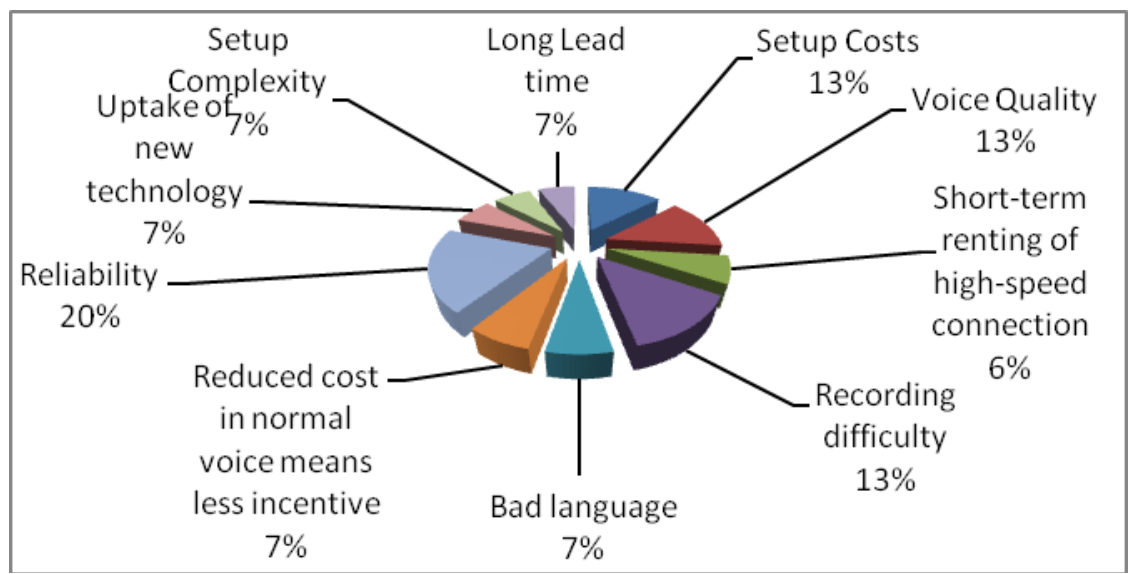

Figure 8 Problems with VoIP as Perceived by the UK Construction Industry

It is interesting to note that problems such as internet connection were raised. Issues of internet connectivity from the construction site are of particular importance, as without a connection the VoIP setup would only be able to provide on-site communications. There are several ways to gain internet connectivity over and above the standard use of a telephone line, which include satellite connections. The advent of WiMAX would also add another method of internet access from the construction site.

If cost savings are the only incentive, then reduced cost of traditional voice calls can render the use of a VoIP system as unnecessary, but the ability to augment different applications together will be lost. This will result in the industry missing out in the opportunities presented by convergence.

It is understandable that the construction industry would have reservations about the technology as it is still in its early adoption phase; however many of the issues are issues that have been addressed to some extent by network vendors.

\section{Convergence to aid construction}

In Australia, the converged network was used to establish telecommunication capabilities to connect remote sites together for Abigroup Limited (Optus, 2005). The setup allowed for communication between two permanent constructions sites whist building a railway line. The setup also provided portable communications facilities that could be torn down at short notice within camps along the construction route as it progressed. This capability is available due to the flexibility provided by IP networks, with the added advantage that the same communications network is use to proved access to data and information.

Several scenarios will be described in this section where the implementation of a converged network can assist the construction project. The majority of improvements lie in benefits gained with immediate access to personnel and information, and the ability to augment different applications together to provide a seamless interface between voice and data. 
Ahsan S, El-Hamalawi, A., Bouchlaghem, D. and Ahmad, S.

The issue of keeping immediate contact with information and personnel is not specific to construction only. In a survey of various businesses (Siemens, 2005), it was found that:

- $\quad 67 \%$ of mobile workers have to leave messages in several different places to actually reach the intended recipient

- $65 \%$ often defer important decisions because their co-workers are unable to respond to their inquiries fast enough

- $59 \%$ are often not able to reach co-workers when they need to speak with them directly on urgent matters

Advantages of converged solutions identified by Siemens include (Siemens, 2005):

- $\quad$ Presence-based communication improves reachability

- $\quad$ Easy organisation of virtual team meetings

- Seamless integration of mobile workers

- Unified communications across all channels

- One number service for easy call forwarding

- Comprehensive consulting services for process optimisation

Different information needs have been identified on the construction site. Research has identified the typical information tasks taking place (De La Garza et al., 1998). Out of the tasks identified, the following were highlighted to have voice as the most important format for information transfer:

- Design and intent clarification

- Contract specification

- Work package information

- Means and methods questions

- Implementation problems

\subsection{Monitoring the design and construction process}

The role of a project manager with regards to this process is as follows (CIB, 1996):

"Monitor design and construction process to see that:

- Adequate and timely information is available to contractor(s) and that their reasonable requests are met

- Production information is available in accordance with programme requirements and that approval of contractors' drawings/specifications is given within the time limits

- Costs are contained within the cost plan/budget

- The overall development objective and detailed programmes are achieved (e.g. meeting progress, specification and quality targets)

- Works supervision and inspection are carried out by parties concerned in accordance with the conditions of their contracts/agreements"

As has already been mentioned, the timely access of information and personnel is important to increasing productivity, as issues can be resolved in minutes rather than hours. The role of a project manager to provide adequate and timely information to the contractor can be effectively supported with the use of a network established on the construction site. The use of a converged network can help further as contact information 


\section{Applications of converged networks in construction}

can be retrieved from a central location ensuring that required personnel are easy to find. Many vendors involved in deploying VoIP products have produced systems that reduce the amount of time it takes to contact people. These systems are called presence servers and are used to identify when a member is contactable and which mode of communication would be best to approach them. This way the chances of contacting the relevant person is increased. Without such a system in place, as identified from other companies by Siemens, project decisions could be delayed until the relevant information or personnel is found, or more dangerous, decisions could be made on insufficient information which can lead to further problems.

The scenario here would require the project manager to carry a PDA, or tablet PC with a soft-phone. Using the device and the wireless network infrastructure connected to the head office via the internet, access to the central store to retrieve data would be carried out as and when information would be needed. Giving all key members of the project team access to devices which allow for voice and data access would provide enhanced collaboration as voice and data information can be transferred simultaneously.

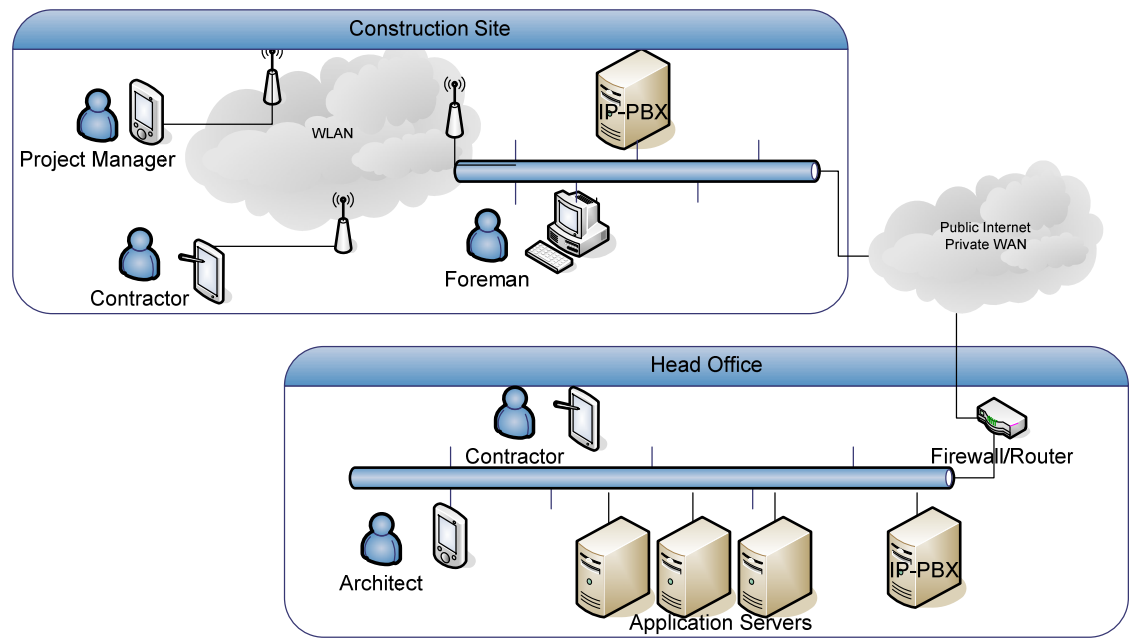

Figure 9 Conceptual Converged Network Deployment

\subsection{Changes in the Client brief}

Construction projects require ongoing changes to solve problems that were unforeseen during the design, to accommodate changes from the client. In order for these changes to be executed, they require communication amongst the relevant personnel as well as written confirmation of the change. Figure 10 shows the overall processes involved in the change request issued by a client. The change request process requires a great deal of communication as well as referencing and updating of project data. This process can become lengthy and impact on the project heavily if the changes are excessive. Applying a converged network can help reduce the time of decision-making by enabling faster access to important data and personnel. 
Ahsan S, El-Hamalawi, A., Bouchlaghem, D. and Ahmad, S.

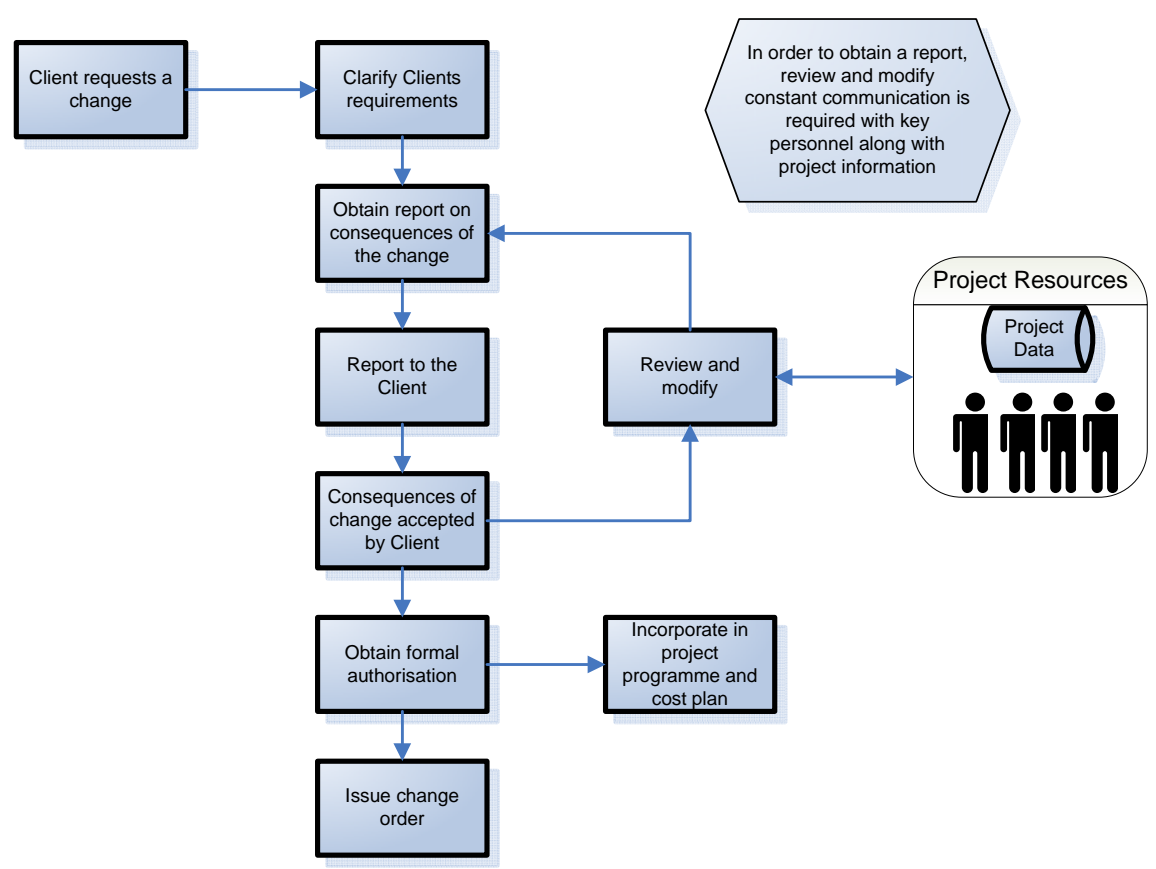

Figure 10 Changes in the client's brief (CIB, 1996)

The process of obtaining a report on the consequences involves access to a number of project members including the project manager, consultant, quantity surveyor and client. Access to project information is essential to make a correct and informed decision, but it is also necessary for the information to be distributed to all necessary individuals in the decision making group. Access to this information onsite can effectively be managed using a network connected to the main headquarters to create what is termed a virtual private network. The virtual private network allows a connection between two separate networks via the internet allowing them to combine as one, with encryption for security. Access to personnel utilising voice over the IP network can allow immediate cost savings for the company. Essentially the process of gathering information can be sped up as there would be less time retrieving relevant information.

Although changes can be requested from the client, there are many changes that are necessary due to other issues that are discovered during construction. When problems occur with the construction varying from initial designs, it is important for the appropriate personnel to be contacted, such as the architect, and decisions made. Since much of this takes place over the phone, when it comes to the project review stage, or there is a dispute regarding the decision, it is important that the change is documented. The process of documenting the change can often be missed due to time constraints, therefore leading to no documentation surrounding the change. The necessity for the conversation to be stored is highlighted by this scenario. The use of converged networks would allow for calls between users to be logged and stored. The saved voice call can either remain as an audio file and be logged along with the rest of the project data, or it can be processed through a speech to text programme which can transcribe the conversation. This transcribed version can therefore be stored along with the rest of the project communication that already exists. The availability of such a file would help in 


\section{Applications of converged networks in construction}

dispute resolution as complete transcripts of conversations and decision taken would be available for inspection.

\section{Conclusion}

The industry as a whole is spending large amounts of money on communication alone. Much of this money is taken up by communication from the construction site to the main office. Wireless networks are being introduced on the construction sites and offices of construction companies. The availability of a network covering a construction site allows for instant access to data around the site. Converging networks, running voice over the same network allows and improves access to personnel as well as data from a site with ease.

The research identified which methods of communication were stored for project review, with voice appearing very low. The complexity of being able to store voice calls would be near impossible due to the voice traffic running on separate networks, but the merging of the two networks into one allows for call logging and recording applications to be easily augmented. The converged network can allow for different applications to be augmented allowing for increased functionality, including voice access to commonly used applications.

The ability to run all communications, data, voice and video through a central network has a number of benefits, the most obvious being of costs and cost control. The ability of instant access to data and personnel can bring increased productivity on site allowing for issues to be resolved quickly ensuring time is not wasted and problems addressed sooner rather than later. Access to call logs can provide detailed information on communication occurring during every stage of a project giving valuable data for the industry to use.

Other advatages offered by convergence include the flexibility to expand the voice and data network easily, the ability to connect different sites together and share resources, the ability to interconnect various applications like SAP to enhance decision making. Of implementations already in place within construction, it seems that the cost saving issue is of paramount importance, however greater benefits can be found with the sharing of resources among various sites giving rise to greater information being available at the time of decision making, thus leading to fewer mistakes being made.

The research has uncovered the benefits of this technology and has shown that it is possible to deploy a setup with the hardware and software currently available. The use of this technology can bring about significant cost savings in the long-run, but the ability to augment different application together to make them accessible to personnel from any location, be it the head office or any of their construction sites, will bring about the greatest benefits.

\section{References}

Agapiou, G., Vali, D., Chatalambakis, D., Georgiadis, P., Plakidis, E., Rorris, P., Ioannou, K., Garmpis, A. "Experimental Performance Evaluation of the emerging WiMAX technology", WSEAS transactions on Information Science and Applications, Vol. 3, Issue 2,2006, pp.322327 
Ahsan S, El-Hamalawi, A., Bouchlaghem, D. and Ahmad, S.

Ahsan, S., El-Hamalawi, A., Bouchlaghem, D., and Ahmad, S. (2005), Using Voice over IP and a Wireless Network to Aid Collaboration in the Construction Industry, Proceedings for CIBW78, 19-21 July, Dresden, Germany, pp 147-154

Ankeny, J. Telephony, Vol. 246, No.12,:Jun 6, 2005, ppG10

Anumba, C. J., Baron, G. and Evbuomwan, N.F.O. "Communications Issues in Concurrent LifeCycle Design and Construction”, BT Technology Journal, Vol.15, No.1, 1997, pp.209-216

AT\&T. “Voice Over IP Comes of Age”, Points of View. 2004.

Beyh, S., and Kagioglu, M. "Construction Sites Communications Towards the Integration of IP Telephony”, ITCon, Vol.9, 2004, pp.325-344

BRE. "BRE guidance on construction site communication", BRE Guidance.( http://projects.bre.co.uk/site_communications/pdf/communication-guidance.pdf) [Accessed 03.03.2005]

Bowden, S. "Mobile Technology Days: The Appropriate Use of I.T. on a Construction Site”, ARUP Publication, 2003

Bowden, S., Dorr, A., Thorpe, A., and Anumba, C. "Mapping site processes for the introduction of mobile IT”, eWork and eBusiness in Architecture, Engineering and Construction, 2004.

CII. “Orinoco Outdoor Wireless LAN”, CII and Purdue University, (http://www.newtechnologies.org/ECT/Other/orinoco.htm) [Accessed 20.09.2004]

Corte A.L., and Sicari, S. "Assessed quality of service and voice and data integration: A case study”, Computer Communications, 2006, Article in Press.

Cus-Babic, N., Rebolj, D., Magdic, A., and Radosavljevic, M. "MC as a Means for Supporting Information Flow in Construction Processes". Concurrent Engineering: Research and Applications 2003;11(1) pp.37-49

Deguine, M., Shulze, F., Leevers, D., Hernandez-Perez, C., Kerber, G., Klaverkamp, W., Camps, M., and Agusti, R. Final Report. ACTS Project AC088 - MICC, Report Number AC088 MICC /WP9/T9200/MICC/99062501/P1.7

De la Garza, J. M., and Howitt, I. "Wireless communication and computing at the construction jobsite”, Automation in Construction, 1998;7 pp327-347

Howard, R., and Petersen, E. “Monitoring Communications in Partnering Projects”, ITCon 2001, Vol.6, pp1-15

IEEE 802.16-2004 Standard. http://standards.ieee.org/cgi-bin/status

IEEE, “Technical Status” (http://grouper.ieee.org/groups/802/15/pub/2003/Mar03/ 03165r0P80215_PC-Wi-Fi-Alliance-PC-update-Mar03.ppt) [Accessed 20.09.2004]

Melinat, B. \& Kelly, B. Evolution of Networks: IP Telephony, available online at: http://www1.worldcom.com/ca/resources/library/reports/ipcomm/wcom_evolution.pdf last updated: 13 April 2004

Meissner, A.; Baxevanaki, L.; Mathes, I.; Branki, C.; Bozios, T.; Schoenfeld, W.; Crowe, M. \& Steinmetz, R. Integrated mobile operations support for the construction industry: the 


\section{Applications of converged networks in construction}

COSMOS solution, International Institute of Informatics and Systemics, Cybernetics and Informatics, 2002.

Miah, T.; Carter, C.; Thorpe, A.; Baldwin, A.N. \& Ashby, S. (1998). Wearable computers: an application of BT's mobile video system for the construction industry, BT Technology, Vol. 16, No. 1.

Optus, "Complete Communications Solution - Abigroup Managed IP Telephony Solution" (http://www.optusbusiness.com.au/convergence/partnership/case-studies_completecommunications.asp) [Accessed 01.04.05]

Prasad, R.V., Hurni, R., Janadagni, H.S., and Shankar, H.N. "Deployment Issues of a VoIP Conferencing System in a Virtual Conferencing Environment”. VRST'03, October 1-3 2003, Osaka Japan

Siemens, "HiPath - "Total Business Communications - Whitepaper, 2005

Shim, Richard. "Specialized markets lead corporate network trend ". CNET News.com. (http://news.com.com/Wireless+wedge/2009-1039_3-5162757.html) [Accessed 01.03.05]

Thus. “A complete guide to Voice over IP”. Guide number TH92767.

Vocera Communications. "Vocera Communications: Bringing Mobile VoIP to In-Building Mobile Workers” (http://www.vocera.com/PDF/Vocera_INB_final_5_5_05.pdf) [Accessed 03.03.05]

Wallingford, Ted. “Switching to VoIP”. O’Reilly Media Inc., 2005. ISBN: 0-596-00868-6

Ward, M., Thorpe, T., Price, A., and Wren, C. "Implementation and control of wireless data collection on construction sites”, ITCon 2004, Vol 9 pp299-311

Wimax.co.uk. "Verso and Saab Grintek Complete VoIP Trials over WiMAX Platform in South Africa” (http://www.wimax.xo.uk/PR2006/JAN2006/2451.htm) [Accessed 17.01.06] 